\title{
The geometry of our universe is definitely FLAT
}

\begin{abstract}
In the nineteen twenties, the famous Russian mathematician Alexander Freedman published a paper on general relativity which revealed that the geometry of our universe can be flat, closed or open. The flat universe obeys the classical Euclidean geometry, the closed universe denotes spherical/elliptic geometry and the open universe is hyperbolic geometry. Recent experimental and observational data show that our universe is flat. In 2019, Anantha Anantha published a paper by delving cosmic triangles that the geometry of our universe is Euclidean. ${ }^{1}$ But till this day, there is no relativistic mathematical verification for flat universe. In this brief note, by analyzing Freedman's modified general relativistic equation, the author attempts to establish that the shape of our universe is definitely flat.
\end{abstract}

Volume 4 Issue 4 - 2020

\section{S Kalimuthu}

Centre for inner studies, India

Correspondence: Sennimalai Kalimuthu, Centre for inner studies, 2/394, Kanjampatti P.O, Pollachi, Tamil Nadu 642003, India,Tel+9| 8220541577, Email owlskaliuthu@gmail.com

Received: June 30, 2020 | Published: July 13, 2020

Keywords: freedman equations, application of algebra, the shape of our universe, relativistic equation, cosmic triangles, curvature parameter, omega greater

\section{Opinion}

\section{Proof ONE}

The density parameter $\Omega$, the curvature parameter k and the Hubble parameter $\mathrm{H}$ are related as ${ }^{1-5}$

$$
(1-\Omega)=-k c^{2} / H^{2} R^{2}
$$

If omega less than $1, \mathrm{k}$ is less than 1 if omega is equal to $1, \mathrm{k}$ is zero If omega greater than $1, \mathrm{k}$ is +1 .

If $\mathrm{k}$ is -1 , the geometry of the universe is open, if it is greater than one, the shape of the universe is closed and the universe obeys Euclidean geometry if $\mathrm{k}$ is equal to zero.

I.e. if $\Omega=1$, the universe is Euclidean, if $\Omega=$ less than 1 , the geometry of the universe is open, and if $\Omega=$ greater than 1 , the universe is closed. For our convenience, let us assume in $(1),-n=-k c^{2} / H^{2} R^{2}$

$$
\text { So, }(1-\Omega)=-n(1 a)
$$

Applying (1a) and cubing (1) we get that, $1-\Omega^{3}-3 \Omega(1-\Omega)=-n^{3}$

i.e. $\left(n^{3}-\Omega^{3)}+1-3 \Omega(1-\Omega)=0\right.$

By applying the famous algebraic cubic formula $a^{3}-b^{3}=(a-b)^{3}+3 a b(a b)$ in the first factor of the above relation we obtain that, $(n-\Omega)^{3}+3 n \Omega(n-\Omega)=-1+3 \Omega(1-\Omega)$

From (1a) we have, $n-\Omega=-1$

Putting this relation in the above eqn. we have, $n(n-\Omega)=\Omega(1-\Omega)(1 \mathrm{~b})$ Again applying (1a) in RHS, $n(n-\Omega)=-n \Omega$ (2) From (1a) we also have, $n-\Omega=-1$

By assuming the above relation in the LHS of (2) we get $-n=-n \Omega$

By simplifying we get that $\Omega=1$

As we have previously noted the shape of our universe is flat if $\Omega$ is equal to one.

\section{ProofTWO}

The density parameter $\Omega$, the curvature parameter $\mathrm{k}$ and the Hubble parameter $\mathrm{H}$ are related as ${ }^{1-5}$

$$
(1-\Omega)=-k c^{2} / H^{2} R^{2}
$$

If omega less than $1, \mathrm{k}$ is less than 1 If omega is equal to $1, \mathrm{k}$ is zero If omega greater than $1, \mathrm{k}$ is +1 .

If $\mathrm{k}$ is -1 , the geometry of the universe is open, if it is greater than one, the shape of the universe is closed and the universe obeys Euclidean geometry if $\mathrm{k}$ is equal to zero.

I.e. if $\Omega=1$, the universe is Euclidean, if $\Omega=$ less than 1 , the geometry of the universe is open, and if $\Omega=$ greater than 1 , the universe is closed,

$$
\text { Let }-n=-k c^{2} / H^{2} R^{2} \text { in (1) }
$$

Assuming (1a) in (2), $1-\Omega+n=0$ Squaring, $1+\Omega^{2}+n^{2}-2 \Omega-2 n \Omega-2 n=0$ i.e. $(\Omega-n)^{2}=2 \Omega+2 n-1=0$

From (1a) we have, $\Omega-n=1$. Putting this in the first factor of the above equation, $1=2 \Omega+2 n-1$

Simplifying, $1=\Omega+n$

But from eqn. (1a), $1=\Omega-n$

Adding the above two relations, $1=\Omega$

As we have previously seen in $\Omega$ is equal to 1 , the curvature of our universe is zero and the geometry of our universe is flat.

\section{Discussion}

The shape of the entire relies on the following properties:

I. Finite or infinite

II. The geometry is flat, hyperbolic or elliptic

III. simply connected space or multiply connected space.

The exact shape is a burning problem in physical cosmology. Several experimental and observational data WMAP, PLANCK, 
BOOMERanG confirm that universe is flat with only a $0.4 \%$ margin of error. Theorists believe that the universe is flat and infinite. Our findings [eqns(3) and (4)] adds more and more favorable arguments for the shape and fate of our universe.

\section{Conclusion}

But still there are problems. Theorists have to determine the global shape of our universe. The global structure of the universes concludes its geometry plus topology. Cosmologists propose various models by using FLRW metric. It will take more and more refinements and advancements to furnish with the complete structures of our universe. Let us recall that the famous French mathematician used to tell time and again that as long as algebra and geometry are not linked into one, we could not expect serious results. Considering this nice quote, the author applied the algebraic cubic formula to Freedman equation to find new result. Also, let us remember Einstein's view. Einstein told that differential equations entered into physics as a maiden servant but became a mistress. Special relativity is purely algebra plus geometry. But that is not the case with general relativity. Einstein wished to deduce many physical results in algebraic system. In this short work, the author attempts to follow both Lagrange's and Einstein's proposals.

\section{Acknowledgments}

None.

\section{Conflicts of interest}

The author declares there is no conflict of interest.

\section{Funding}

None.

\section{References}

1. Hedgcock J. Toward a socioliterate approach to second language teacher education. Modern Language Journal. 2020;86:299-317.

2. Anantha Anantha. On the geometry of our universe. New Trends in Mathematical Sciences. 2019;715-21.

3. Gomerro GI. Determining the shape of the Universe using discrete sources. Class Quant Grav. 2003;20:4775-4784.

4. R Aurich, S Lustig, F Steiner, et al. Can one hear the shape of the universe. Phys Rev Letts. 2005;94:021301-1 - 021301-4.

5. Neil Cornish, David Spergel, Glenn Starkman. Can COBE see the shape of the universe? Phys Rev D. 1998;57:5982-5996.

6. Gomerro GI. Can We See the Shape of the Universe? Int $J$ Mod Phys. 2002;17:4281-4286. 